\title{
Fetal growth velocity: kinetic, clinical, and biological aspects
}

Neonatal Unit, University of Torino E Bertino C Fabris

Institute of

Puericulture and

Neonatal Medicine L

Gaslini, University of Genova

E Di Battista

G Aicardi

Institute of Medical Statistics and

Biometry, University of Milano

A Bossi

S Milani

Department of Obstetrics and Gynaecology, University of Torino M Pagliano

Correspondence to: Anna Bossi, Istituto di Statistica Medica e Biometria, Università di Milano, Via Venezian 1 I-20133 Milano, Italy.

Accepted 9 August 1995 Enrico Bertino, Eliana Di Battista, Anna Bossi, Marco Pagliano, Claudio Fabris,
Giorgio Aicardi, Silvano Milani

\begin{abstract}
With the aim of determining fetal growth kinetics, prenatal data were analysed which had been longitudinally collected in the framework of a perinatal growth survey. The sample comprised 238 singleton normal pregnancies, selected in Genoa and Turin (between 1987 and 1990), and repeatedly assessed by ultrasound scans (five to nine per pregnancy). Five morphometric traits were considered: BPD (biparietal diameter), OFD (occipitofrontal diameter), HC (head circumference), FDL (femur diaphysis length) and AC (abdomen circumference).

Growth rate seemed to increase in the early part of the second trimester, and decrease subsequently: velocity peaks were steeper and earlier for head diameters and circumference (about 18 weeks) than for femur length (20 weeks) and abdomen circumference ( 22 weeks). Velocity standards were traced using a longitudinal two-stage linear model: this ensures unbiased description of the shape of the growth curve, even when growth kinetics are asynchronous, and efficient estimation of the outer centiles - the most useful for diagnostic purposes.

(Arch Dis Child 1996; 74: F10-F15)
\end{abstract}

Keywords: fetal growth velocity, head diameters, head and abdomen circumferences, femur length.

Before the development of ultrasonography, there was no harmless and reliable technique available for providing information on physiological fetal growth kinetics, and the so-called intrauterine growth standards were therefore based on anthropometric measures of neonates with different gestational ages. As the assumption that prenatal growth in full term and preterm babies follows the same pattern is hardly tenable, ${ }^{1}$ these norms are intrinsically a tool for evaluating body size and proportion of neonates, and not true growth standards for monitoring fetal development. ${ }^{2}$

Unfortunately, most ultrasonographic norms derive from cross-sectional surveys, ${ }^{3-5}$ or from longitudinal surveys involving either a limited number of observations per pregnancy or a limited number of pregnancies. ${ }^{6-10}$ Even in the case of longitudinal studies, data are generally processed as coming from crosssectional surveys, so that the specific possibilities of these studies (such as the estimation of growth velocity and the analysis of the pattern of growth) are overlooked and lost.
In this paper we present a new kinetic model for fetal growth and velocity charts computed on a large sample of pregnancies, collected and repeatedly assessed by ultrasound scans in the framework of a prospective perinatal growth longitudinal survey.

\section{Methods}

Pregnant women were selected in Genoa and Turin, between 1987 and 1990, in accordance with the restrictive criteria recommended by FIGO, ${ }^{11}$ so as to include only normal singleton pregnancies. The sample so obtained comprised 238 neonates (123 girls, 115 boys) delivered at term after low risk, uncomplicated pregnancy. Gestational age was estimated from the last menstrual period and was confirmed by an early ultrasound assessment of crown-rump length ${ }^{12}$ performed within the 12th week: when the difference between the two estimates exceeded seven days, the pregnancy was excluded from the reference set. Informed consent was obtained from each woman participating in the survey.

Individual growth profiles consist of five to nine measures (totalling from 1539 scans for biparietal diameter, to 1237 scans for abdomen circumference), taken between the 12th and the 40th gestational week. All measurements were performed by four trained echographers, using real time scanners (Ansaldo AU920, ATL Ultramark IV, Toshiba SAL 30A) equipped with $3.5 \mathrm{MHz}$ transducers. In accordance with the method described by Campbell, ${ }^{13}$ BPD (biparietal diameter) was measured at the largest transverse diameter of head at the level of thalamus, from the outer edge of the proximal skull table to the inner edge of the distal skull tables, whereas OFD (occipitofrontal diameter) was obtained from middle to middle measurements on the same plane used for BPD. ${ }^{14} \mathrm{HC}$ (head circumference) was computed from the short and long axes of head taken from the outer contour of skull profile. ${ }^{15}$ FDL (femur diaphysis length) was determined as the largest of three measures obtained according to Queenan et al. ${ }^{16} \mathrm{AC}$ (abdomen circumference) was computed from the antero-posterior and transverse abdomen diameters, measured as two perpendicular axes of the outer contour of abdomen profile at the level of the median portion of porto-umbilical vein passing through the liver, as indicated by Kurjac and Breyer. ${ }^{17}$

A test-retest experiment was performed to estimate the comparability of measurements. A set of 20 pregnant women with pregnancies 


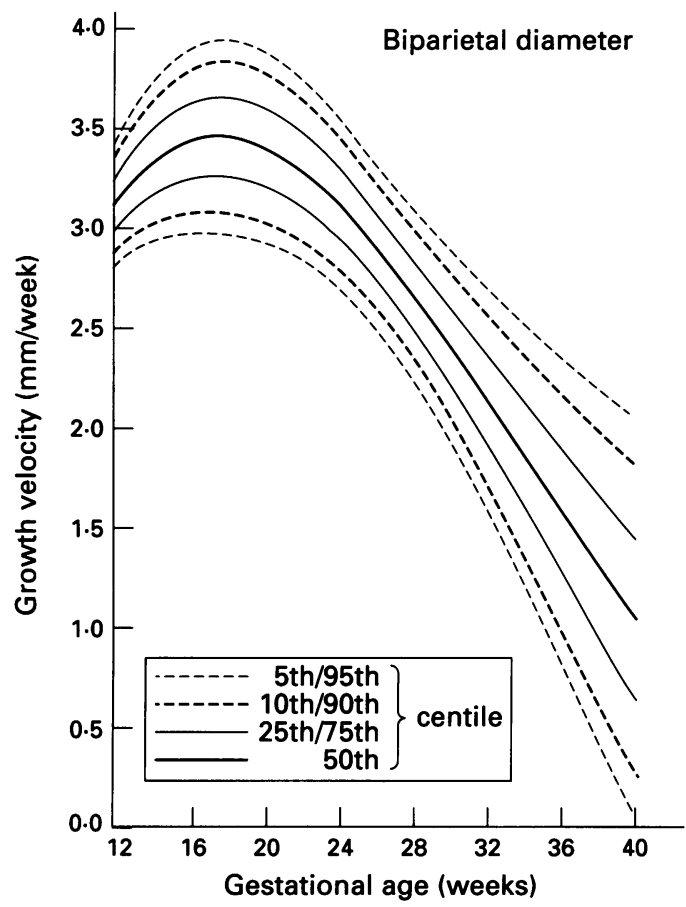

Figure 1 Standards of BPD (biparietal diameter) growth velocity ( $\mathrm{mm} /$ week) as a function of menstrual age

(weeks). The chart applies to estimates of growth velocity based on measures taken six weeks apart.

ranging from 13 to 36 weeks (the same number of pregnancies in the second and in the third trimesters), were independently examined by two echographers. The average measuring errors $(\mathrm{mm})$, expressed as $s_{\text {means }}$ and concerning the second and the third trimester, respectively, were as follows: 0.67 and 0.76 (BPD), 1.26 and 1.46 (OFD), 2.43 and 3.49 (HC), 0.74 and 0.88 (FDL), 4.02 and 6.44 (AC).

\section{STATISTICAL METHODS}

\section{Growth functions}

Most of the mathematical functions used to model prenatal growth of BPD and other one dimensional traits belong to the same family of three constant linear growth functions:

$$
\log _{\mathrm{e}}\left\{\mathrm{y}_{\mathrm{i}}(\mathrm{t})\right\}=\alpha_{0 \mathrm{i}}+\alpha_{1 \mathrm{i}} f_{1}(\mathrm{t})+\alpha_{2 \mathrm{i}} f_{2 \mathrm{i}}(\mathrm{t})+\epsilon_{\mathrm{i}}(\mathrm{t})
$$

Where $y_{i}(t)$ is the value of the trait measured on the $\mathrm{i}$-th individual at age $\mathrm{t} ; \epsilon_{\mathrm{i}}(\mathrm{t})$ is the intraindividual random term associated with $\log _{\mathrm{e}}\left\{\mathrm{y}_{\mathrm{i}}(\mathrm{t})\right\} ; \alpha_{0 \mathrm{i}}, \alpha_{1 \mathrm{i}}$, and $\alpha_{2 \mathrm{i}}$ are the growth constants of the $\mathrm{i}$-th individual; lastly $f_{1}(\mathrm{t})$ and $f_{2}(t)$ are selected functions of age which determine the shape of growth model. If we set $f_{1}(\mathrm{t})=\mathrm{t}$ and $f_{2}(\mathrm{t})=\log _{\mathrm{e}}(\mathrm{t})$ we get a Count function $^{18}$ in a log scale (we shall call it log count function). The logarithmic form of RossavikDeter ${ }^{19}$ and Todros et $\mathrm{al}^{4}$ exponential-power functions may be derived by log-Count function if we set $f_{1}(\mathrm{t})=\mathrm{t} \times \log _{\mathrm{e}}(\mathrm{t})$ and $\alpha_{2}=2$, respectively. All these functions have a point of inflection ${ }^{419}$ but do not have an upper horizontal asymptote, thus allowing for the fact that fetal growth velocity increases moderately in the first months of gestation, and then decreases slowly without falling to zero. ${ }^{20}$

The comparison of the functions mentioned above on the basis of the analysis of residuals and of the precision of the estimates indicated that Todros et al (for AC) and log-Count functions (for the remaining traits) led to the most satisfactory description of fetal growth: the maximum systematic departure of these functions for the growth profile was less than $3 \% .^{21}$ The analysis of fetal growth kinetics and velocity standards presented are based on these functions.

Two-stage models and longitudinal standards The fitting of appropriate functions to individual growth profiles may be regarded as the first stage of an explicit two-stage model. ${ }^{22}$ In this first stage the individual growth constants were estimated by ordinary least squares method, under the usual assumptions that the intra-individual component of variance (which accounts for both measurement error and biological random fluctuations due to variations of the intrauterine environment and to nutritional factors) is nearly the same over time and subjects, and that repeated measures are uncorrelated. In the case of intrauterine growth these assumptions seem to be sensible and fairly consistent with empirical evidence, provided that a log scale is adopted, homogeneous reference groups are selected, and a flexible growth function (such as the logCount function defined here) is fitted to data. $^{21}$

In the second stage the average growth constants (which express the mean growth pattern of the reference population) and the interindividual covariance matrix (which expresses differences between the individual growth patterns of fetuses belonging to the same reference group) were simultaneously estimated by an expectation maximisation interactive algorithm (Milani S, Bossi A, Marubini E. Paper contributed to 46th Session of International Statistical Institute, Tokyo, 1987) which leads to generalised least squares estimates. This method allows for the fact that individual growth constants are estimated from observations which differ in number and time location.

Growth velocity charts (the centiles of the distribution of the growth velocity of a given trait conditional on gestational age) were derived from the above average growth constants and interindividual covariance matrix, under the hypothesis that the measures are taken six weeks apart for BPD, OFD, HC, and FDL, and 10 weeks apart for AC: because of measurement errors, velocity values computed on shorter time intervals are too unreliable to be of practical use (see the appendix for the algebraic details).

\section{Results}

Figures 1-5 show growth velocity standards for BPD, OFD, HC, FDL, and AC. For all these traits, growth velocity seemed to increase in the early part of the second trimester and decrease subsequently up to the end of pregnancy. Interindividual variability of growth velocity changes remarkably with gestational age: it 
Figure 2 Standards of $O F D$ (occipitofrontal diameter) growth velocity $(\mathrm{mm} / \mathrm{week})$ as a function of menstrual age (weeks). The menstrual age (weeks). The
chart applies to estimates of growth velocity based on measures taken six weeks apart.

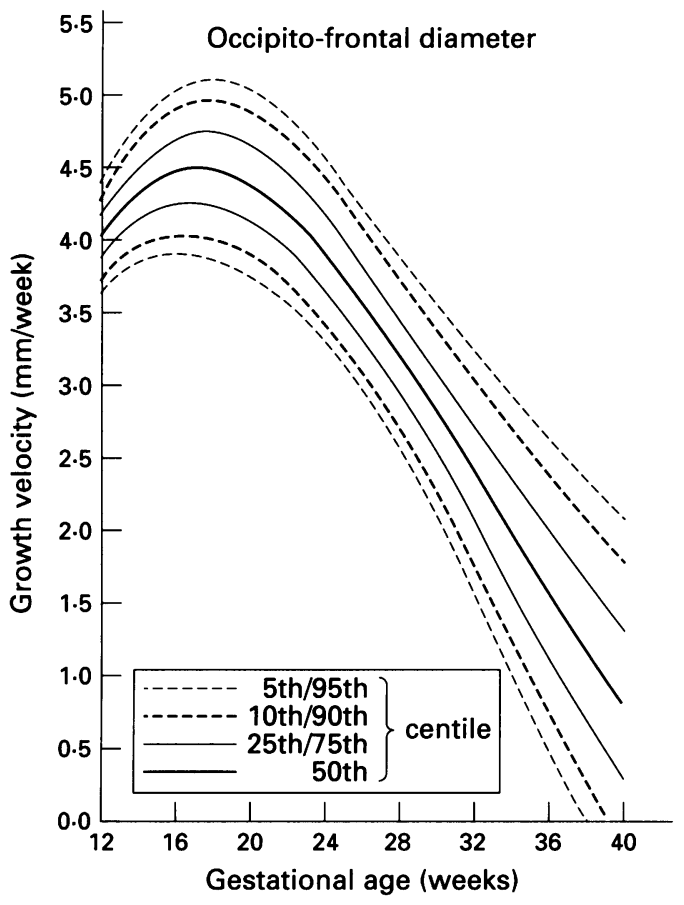

rises up to the peak of velocity, lessens reaching a minimum between 25 and 30 weeks of gestation, and then increases again up to the end of the pregnancy.

Different morphometric traits display different ages at the peak of growth velocity. The peak of velocity occurs earlier in head measurements (BPD: 17.9 completed weeks (SE 0.15), OFD: $17 \cdot 4 \quad(0 \cdot 13)$, HC: $17 \cdot 3$ $(0 \cdot 11))$, and later for FDL $(20 \cdot 2(0 \cdot 13))$ and AC $(22 \cdot 1(0 \cdot 27))$. Furthermore, the change of velocity with age is less for AC than for head diameters and circumference, while is somewhat more prominent in FDL. The above

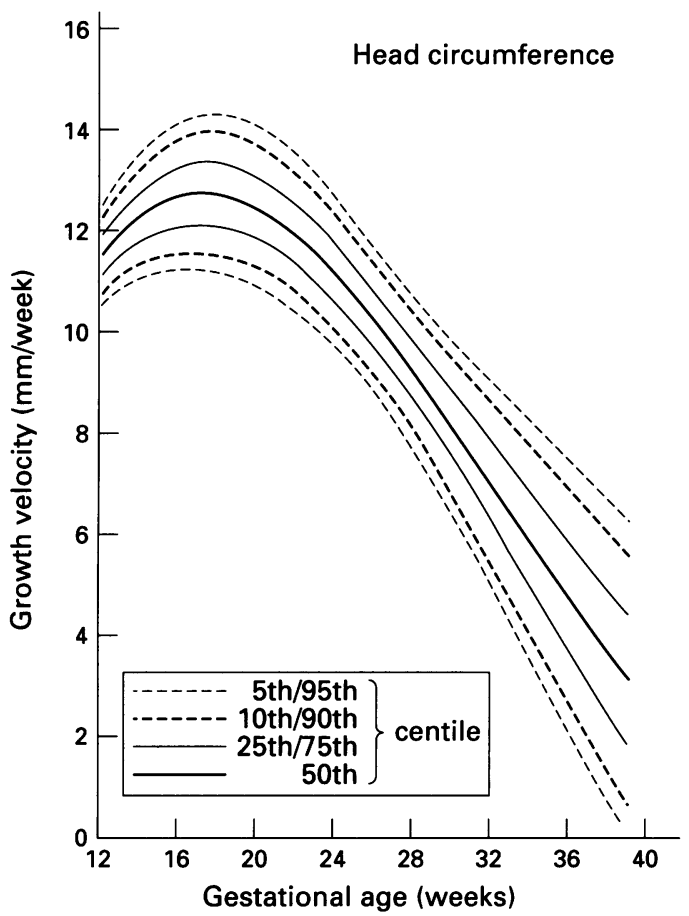

Figure 3 Standards of HC (head circumference) growth velocity ( $\mathrm{mm} /$ week) as a function of menstrual age (weeks). The chart applies to estimates of growth velocity based on measures taken six weeks apart. differences in the pattern of growth of the traits under study are shown in figure 6 , where the velocity of each trait is expressed as a percentage of its size at 40 gestational weeks. In the same figure the growth velocity of cubic BPD (which is roughly proportional to head volume) is also shown: the peak velocity is sharper and takes place much later $(31 \cdot 1$ weeks $(0 \cdot 27)$ ) for computed head volume than for head diameters and circumference.

Only minor differences in the growth pattern of one dimensional traits emerged between sexes: differences seemed to be negligible from a clinical point of view and could be ignored in drawing prenatal growth velocity charts.

\section{HEAD}

The estimated value of gestational age at peak of velocity was about 17-18 weeks for BPD, $\mathrm{OFD}$, and HC. Lower values were derived from the mixed longitudinal study (13-16 weeks) of Guihard-Costa $e t a l^{10}$ and from the cross-sectional study (16.4 weeks) of Todros et $a l .{ }^{4}$ Higher values $(20.8$ weeks) are reported by Erikssen et al $^{23}$ on the basis of a longitudinal study. Similar velocity values and analogous decrease of velocity after the 17th gestational week were observed by Campbell et $a l^{6}$ in a longitudinal study (191 pregnancies, 3.4 scans/pregnancy): however, in their study the age at peak could not be estimated, because of the lack of measurements before the 17 th week. Jeanty et al $^{24}$ and Munjanja et al ${ }^{25}$ observed that growth velocity increases after 14-15 gestational weeks. From the longitudinal observation of 30 pregnancies, Fescina et $a l^{26}$ infer that the maximum growth velocity of intracranial perimeters occurs between the 14 th and the 16th gestational weeks. Deter et $a l,{ }^{27}$ in the first longitudinal study aimed to model fetal growth, and which involved only 20 pregnancies, observed that BPD growth curve has a slight upward convexity which continues up to 38 weeks of gestation, whereas $\mathrm{HC}$ is characterised by a linear phase up to 30 weeks and by a subsequent slow down in growth velocity. Ten years later, Deter and Harrist $^{28}$ observed that HC growth rate decreases from $14 \mathrm{~mm} /$ week (at 14 weeks of gestation) to $9 \mathrm{~mm} /$ week (at 30 weeks) and $5 \mathrm{~mm} /$ week (at 38 weeks).

Our data indicate that peak growth velocity for BPD, OFD, and HC occurs at the end of the first phase of rapid increase in forebrain cell number, when major neuronal proliferation occurs. The subsequent decrease in growth velocity of these one dimensional traits does not necessarily reflect a contemporary slow down in the weight gain of the brain (which is a complex three dimensional structure and approximates the growth pattern of cubic BPD), whose peak growth velocity seems to be later than those of one dimensional traits. ${ }^{29}$ Our findings agree with those of Meire, ${ }^{30}$ who points out that the growth curve of fetal head volume has a shape rather different from that of BPD, with a velocity decrease after 28-32 weeks. 
Figure 4 Standards of FDL (femur diaphysis length) growth velocity ( $\mathrm{mm} /$ week) as a function of menstrual age (weeks). The chart applies to estimates of growth velocity based on measures taken six weeks apart.

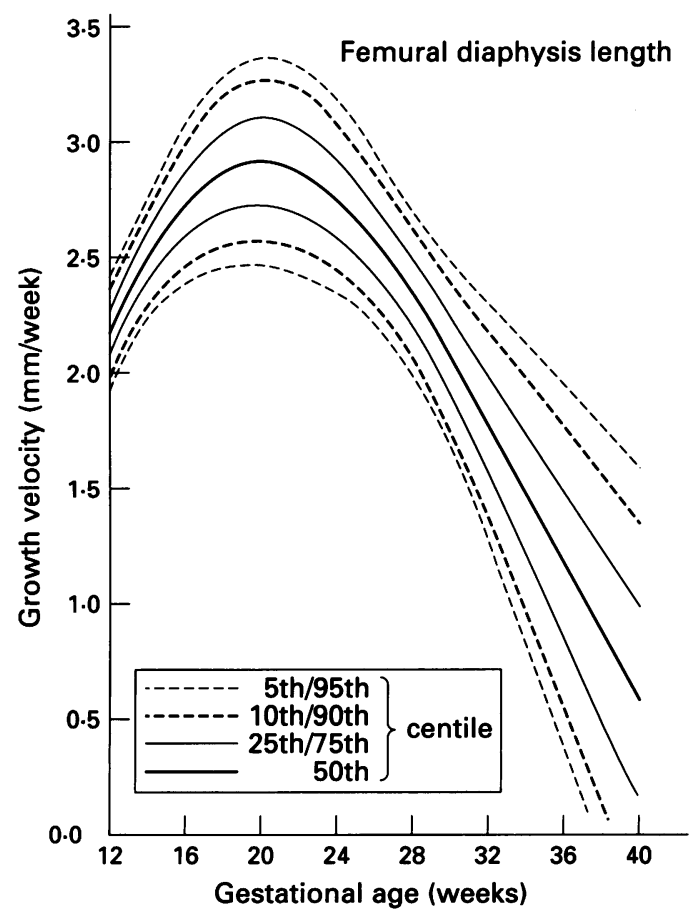

FEMUR

At present there are relatively few data suitable for modelling the growth kinetics of the femur. On the basis of longitudinal observations, Queenan et $a l^{7}$ state that growth is linear between 12 and 22 weeks of gestation. In a more recent study Exacoustos et al $^{5}$ notice that growth is fairly linear from 13 to 28 weeks and then slows down, even if growth velocity cannot be estimated from their cross-sectional data. Cross-sectional data reported by Yeh et $\mathrm{al}^{3}$ and longitudinal observations carried out by Elejalde and De Elejalde, ${ }^{9}$ suggest that growth of the femur is linear between 10 and 40 weeks of gestation: but such a pattern seems to be unusual for any biological growth process. ${ }^{31}$ Longitudinal data reported by O'Brien et al ${ }^{8}$ and Brons et $a l^{32}$ display similar growth patterns with a decrease in velocity after 17 and 12 weeks of gestation, respectively. Only Guihard-Costa et $a l,{ }^{10}$ on the basis of mixed longitudinal data, observe a non-monotonic kinetic pattern, with a peak between 13 and 16 weeks and a subsequent decrease in velocity.

From our longitudinal study it emerged that FDL growth velocity peaks at about 20 weeks when, according to Tanner, ${ }^{33}$ the peak of velocity in fetal crown-heel length occurs, which is highly correlated with femur length.

\section{ABDOMEN}

As to growth of abdomen circumference, the age at peak estimated from our study (22 weeks) is higher than those reported by Guihard-Costa et al ${ }^{10}$ (from 13 to 16 weeks) and Todros et $\mathrm{al}^{4}$ (20 weeks), but lower than that given by Fescina et $\mathrm{al}^{26}$ (from 32 to 34 weeks). From a longitudinal analysis of 20 pregnancies Deter et $a l^{27}$ concluded that AC growth is essentially linear throughout pregnancy. Ten years later, Deter and Harrist ${ }^{28}$ asserted that AC growth velocity decreases slightly: from $12 \mathrm{~mm} /$ week (at the 14th week of gestation) to $11 \mathrm{~mm} /$ week (by the 38 th week).

Our analysis indicates out that the variations in $\mathrm{AC}$ growth velocity during prenatal life are less prominent than those of head and femur, which agrees with the findings of Erikssen et al ${ }^{23}$ and Meire, ${ }^{30}$ who remark that AC growth is more linear than that of the other traits. AC reflects, to a large extent, the size of the liver and the thickness of subcutaneous fat tissue which grow mainly in the last trimester of pregnancy, ${ }^{34} 35$ and is reported as a trait useful to estimate fetal weight. ${ }^{17}$ However, the peak of AC growth velocity comes far earlier than that of body weight, which is supposed to occur between 34 and 38 weeks of gestation. ${ }^{33}$ This time lag may be explained by several factors.

First, one dimensional traits progress arithmetically throughout gestation, while weight, which is proportional to volume, progresses geometrically, rather like cube BPD. Secondly, abdomen circumference mainly reflects the transversal size of the liver, and not its volume, whose exponential growth is similar to that of body weight. ${ }^{36}$ Finally, more subcutaneous fat, which contributes predominantly to body weight during the third trimester of pregnancy, is stored in limbs and subscapular sites than in the abdomen. ${ }^{37}$

\section{DIFFERENCES BETWEEN FETAL GROWTH} KINETICS

In our study the peak of $\mathrm{AC}$ growth velocity occurred just after the end of adipose tissue appearance in the abdominal wall where this happens earlier than in the limbs. ${ }^{38}$ At variance with the findings outlined by Guihard-Costa et $a l,{ }^{10}$ in our study a prominent difference emerged between the ages at peak velocity for head, femur, and abdomen. In fact, it is widely

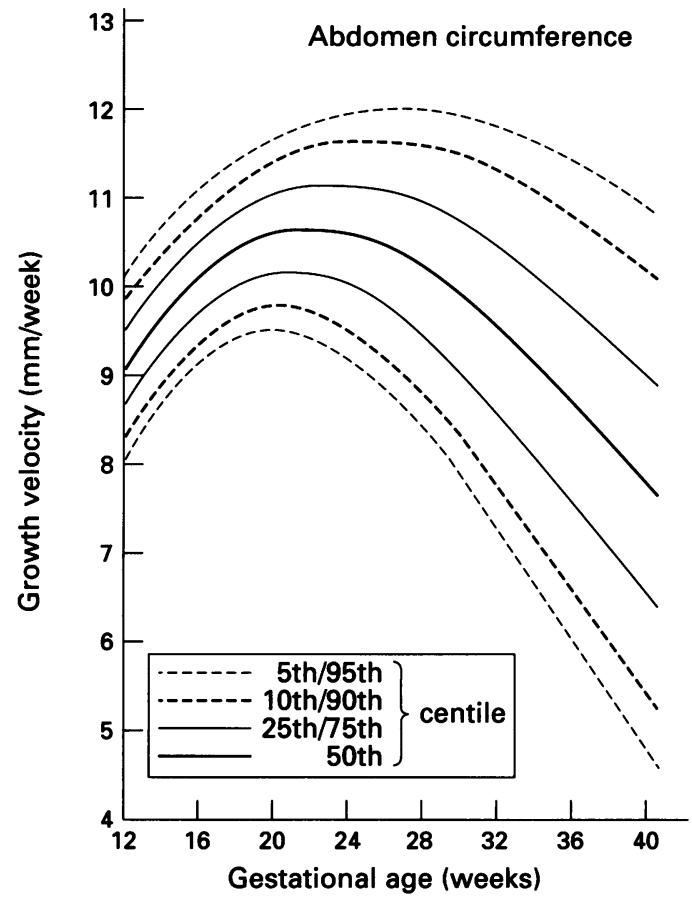

Figure 5 Standards of $A C$ (abdomen circumference) growth velocity $(\mathrm{mm} /$ week) as a function of menstrual age (weeks). The chart applies to estimates of growth velocity based on measures taken 10 weeks apart. 
Figure 6 Mean growth velocity (as per cent of the size attained at 40 weeks) as a function of menstrual age (weeks). Head volume (HV) was regarded as proportional to cubic BPD.

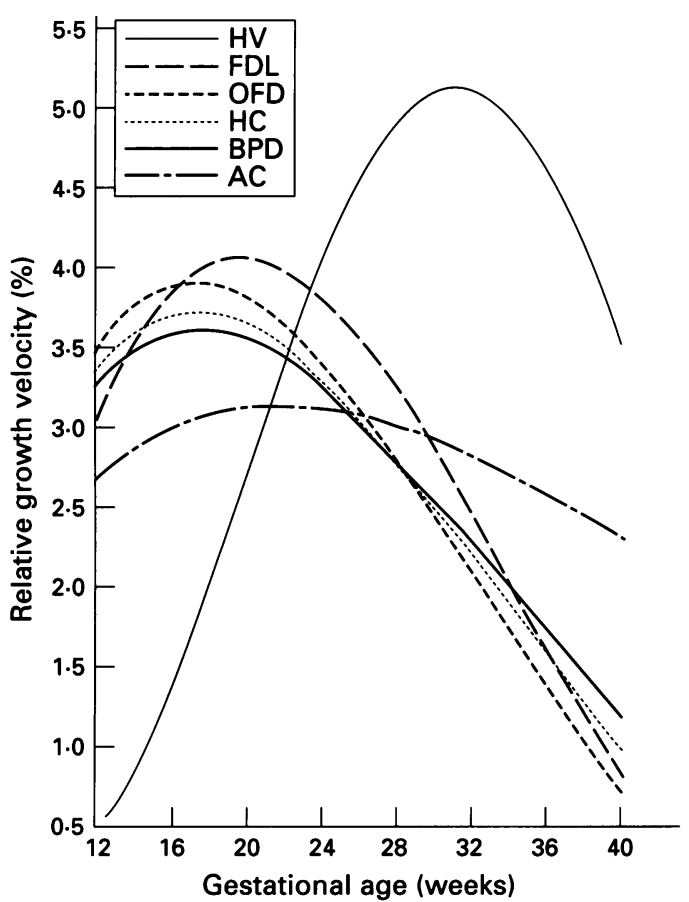

known that fetal growth is characterised by different rates for organ weight and size, limb length and head circumference and volume, so that body shape and proportions change over gestation. ${ }^{39}$ On the other hand, differences in fetal growth kinetics of head and abdomen circumferences emerge also from the inspection of preterm and full term babies: preterm neonates have a ratio of head and brain size to body mass that is larger than that of full term neonates, and this may account for their very high glucose turnover. ${ }^{40}$ Anoxia affecting fetal development in the first four months of gestation results in generalised growth impairment (weight, length, and head size), while anoxia occurring in the last months of pregnancy affects mainly weight and results in a low birthweight baby with normal head circumference and length. ${ }^{41}$

\section{Discussion}

Intrauterine growth velocity has a nonmonotonic pattern: it increases in the first part of pregnancy and decreases in the last part. The age at peak velocity as well as the extent of the peak depend on the morphometric traits: peaks are sharper and occur later for three dimensional traits than for one dimensional traits. Furthermore, abdomen circumference reaches maximum growth velocity two weeks after femur length and five weeks after head circumference. The reliability of these conclusions rests on the number of records per longitudinal profile (from five to nine, mean $=6 \cdot 5)$, the size of sample (238 pregnancies), the strictness of criteria adopted to select normal pregnancies resulting in normal outcome, and the relatively good precision of ultrasonographic measurements.

The velocity standards presented here are entirely based on a two-stage linear model. In the first stage the individual intrauterine growth profiles were fitted by a proper growth function: Todros et al 's $\mathrm{s}^{4}$ function for $\mathrm{AC}$ and a new function derived from Count's function ${ }^{18}$ for the other fetal traits (log-Count function). The systematic discrepancies which emerged between observed and fitted values (less than $3 \%$ ) are negligible from a clinical viewpoint. The second stage implied the estimate of the mean constant curve and of the covariance matrix which expresses interindividual variability, required to compute growth centiles. Using this method, the shape of velocity curve can be described without bias, even when growth kinetics are not synchronous among individuals, and efficient estimates are attained even for the outer centiles which are the most useful for diagnostic purposes. Unfortunately, the precision of the current ultrasonographic techniques is still inadequate to obtain reliable measures of individual growth velocity on short time intervals. This drawback restricts the practical use of velocity charts to velocity estimates derived from measures taken at least six (for BPD, OFD, HC, and FDL) or 10 (for AC) weeks apart.

\section{Appendix}

Let us consider a morphometric trait (y) whose expected growth in a given subject is described by a continuous function of time $(t)$. This function is characterised by a vector of individual parameters $\alpha$ which, in the population from which the subject has been drawn, is a random variable with mean vector $\beta$ and covariance matrix $\underline{\Sigma}_{\alpha}$ :

$$
\mathrm{E}(\mathrm{y} \mid \underline{\alpha})=f\{\underline{\alpha} ; \mathrm{t}\} \quad \mathrm{E}(\mathrm{y})=f\{\underline{\beta} ; \mathrm{t}\} \quad \mathrm{V}(\underline{\alpha})=\underline{\Sigma}_{\alpha}
$$

Therefore, a single measure y taken on the subject at time t may be expressed as

$$
\mathrm{y}=f\{\underline{\alpha}, \epsilon ; \mathrm{t}\}=f\{\underline{\beta},(\underline{\alpha}-\underline{\beta}), \epsilon ; \mathrm{t}\}
$$

where random terms $(\alpha-\beta)$ and $\epsilon$ account for interindividual and intra-individual variability. The fetal growth models (log-Count function and Todros et $a l^{4}$ functions) on which standards here presented are based may be written as:

$$
y=\exp \left\{\underline{q^{\prime}} \underline{\beta}+\underline{q}^{\prime}(\underline{\alpha}-\underline{\beta})+\epsilon\right\}
$$

where $\mathrm{q}^{\prime}=\left\{1, \mathrm{t}, \log _{\mathrm{e}}(\mathrm{t})\right\}$. Under the usual assumption for random terms (ie, $V(\epsilon)=\sigma^{2}$, $\operatorname{Cov}\left(\epsilon_{\mathrm{i}}, \epsilon_{\mathrm{j}}\right)=0$, and $\operatorname{Cov}\left\{\mathrm{q}^{\prime}(\underline{\alpha}-\underline{\beta}), \epsilon\right\}=0$, the variance of a new observation ( $\tilde{y})$ at time $t$, predicted on the basis of an unbiased estimate $\hat{\beta}$ of $\beta$, is given by the sum of the interindividual and intra-individual components, ${ }^{42}$ and may be computed (approximately) from equation 3 by means of a Taylor series expansion about $f\{\beta ; \mathrm{t}\}$

$$
\mathrm{V}(\tilde{\mathrm{y}}) \cong[f\{\underline{\beta} ; \mathrm{t}\}]^{2} \times\left\{\left[\mathrm{q}^{\prime}\left(\underline{\Sigma}_{\alpha}+\mathrm{V}(\underline{\hat{\beta}})\right) \underline{\mathrm{q}}\right]+\sigma^{2}\right\}
$$

Let us denote the first derivative of vector $\mathrm{q}^{\prime}$ respect to time $t$ as $\partial q^{\prime}=\{0,1,1 / t)$ and write the expected individual growth velocity as:

$$
\mathrm{E}(\mathrm{v} \mid \underline{\alpha})=\partial f\{\underline{\alpha} ; \mathrm{t}\} / \partial \mathrm{t}=f\{\underline{\alpha} ; \mathrm{t}\} \times\left\{\partial \underline{q}^{\prime} \underline{\beta}+\partial \underline{\mathrm{q}}^{\prime}(\underline{\alpha}-\underline{\beta})\right\}
$$


The variance of a predicted value of velocity $\tilde{v}$ is given by the sum of an interindividual component, which does not depend on the time interval $\Delta t$ on which velocity is measured, and of an intra-individual component which does depend on $\Delta \mathrm{t}$ :

$$
\mathrm{V}(\tilde{\mathrm{v}}) \cong \mathrm{V}(\mathrm{E}(\tilde{\mathrm{v}} \mid \underline{\alpha}))+[f\{\underline{\beta} ; \mathrm{t}\}]^{2} \times 2 \sigma^{2} / \Delta \mathrm{t}^{2}
$$

where $\mathrm{V}(\mathrm{E}(\tilde{\mathrm{v}} \mid \alpha))$ may be computed (approximately) from equation 5 by means of a Taylor series expansion about $f\{\beta ; \mathrm{t}\}$

$\mathrm{V}(\mathrm{E}(\tilde{\mathrm{v}} \mid \underline{\alpha})) \cong[f\{\hat{\beta} ; \mathrm{t}\}]^{2} \times\left\{\left(\partial \mathbf{q}^{\prime} \underline{\beta} \beta^{\prime} \partial \mathrm{q}\right) \times\left[\mathbf{q}^{\prime}\left(\underline{\Sigma}_{\alpha}+\mathrm{V}(\hat{\beta})\right) \mathrm{q}\right]+\right.$
$\left.+\left[\partial \underline{q}^{\prime}\left(\underline{\Sigma}_{\alpha}+\mathrm{V}(\underline{\hat{\beta}})\right) \underline{q}\right]+2 \times\left(\partial \underline{q}^{\prime} \underline{\beta}\right) \times\left[\underline{q}^{\prime}\left(\underline{\Sigma}_{\alpha}+\mathrm{V}(\underline{\hat{\beta}})\right) \underline{q}\right]\right\}$

The estimate of $\mathrm{V}(\tilde{\mathrm{v}})$ was obtained by replacement of $\underline{\beta}, \Sigma_{\alpha}, V(\hat{\beta})$ by their generalised least square estimates $\hat{\beta}, \hat{\Sigma}, \hat{\mathrm{V}}(\hat{\beta})^{22}$, and $\sigma^{2}$ by the pooled residual mean square error $\left(\mathrm{s}^{2}\right)$ about the individual growth curves.

For all traits, the distribution of the fitted values of individual growth velocities conditional on gestational age was close to the normal distribution: this is not surprising, as all fetal traits under study are one dimensional. For this reason, the usual parametric estimates of centiles were computed. As $\Delta t$, we chose an interval of six weeks for BPD, OFD, $\mathrm{HC}$, and FDL and an interval of 10 weeks for AC, the measures of which seemed to be affected by relatively larger errors. The choice was a compromise between the size of technical error and the obstetrician's need to have a ready assessment of the normality of fetal growth.

We are deeply grateful to Professors J M Tanner and M J R Healy, whose valuable experience and incisive suggestions supplied us with insight into the methodological aspects implied in the construction of velocity standards.

Supported in part by Grants from Piemonte and Liguria Supported in part by Grants from Piemo
regions, and from Istituto G Gaslini, Genova.

regions, and from Istituto G Gaslini, Genova.
Presented in part (as an abstract) at the 6th International Presented in part (as an abstract) at the 6th
Congress of Auxology, Madrid, September 1991.

1 Falkner F. Perinatal growth. Arch Franç Pédiatr 1985; 42: 195-7.

2 Secher NJ, Kern Hansen P, Thomsen BL, Keiding N. Growth retardation in preterm infants. $\mathrm{Br} \mathcal{f}$ Obstet Gynaecol 1987; 94: 115-20.

3 Yeh MN, Bracero L, Reilly KB, Murtha L, Aboulafia M, Barron BA. Ultrasonic measurement of the femur length as an index of fetal gestational age. Am $¥$ Obstet Gynecol as an index of fetal

4 Todros T, Ferrazzi E, Groli C, Nicolini U, Parodi L, Pavoni $M$, et al. Fitting growth curves to head and abdomen measurements of the fetus: a multicentric study. $\mathcal{f}$ Clin Ultrasound 1987; 15: 95-105.

5 Exacoustos C, Rosati P, Rizzo G, Arduini D. Ultrasound measurements of fetal limb bones. Ultrasound Obstet Gynecol 1991; 1: 325-30.

6 Campbell S, Newman GB. Growth of the fetal biparietal diameter during normal pregnancy. $\mathcal{f}$ Obstet Gynaecol $\mathrm{Br}$ Cwlth 1971; 78: 513-9.

7 Queenan JT, Kubarych SF, Cook LN, Anderson GD, Griffin LP. Diagnostic ultrasound for detection of intrauterine growth retardation. Am $\mathcal{f}$ Obstet Gynecol intrauterine growth

8 O'Brien GD, Queenan JT. Growth of the ultrasound fetal femur length during normal pregnancy. Am $\mathcal{f}$ Obstet Gynecol 1981; 141: 833-7.

9 Elejalde BR, de Elejalde MM. The prenatal growth of the human body determined by the measurement of bones and organs by ultrasonography. Am $\mathcal{f} M e d$ Genet 1986; 24: 575-98.

10 Guihard-Costa AM, Droulle P, Larroche JC. Growth velocity of the biparietal diameter, abdominal transverse diameter and femur length in the fetal period. Early Hum Dev 1991; 27: 93-102.

11 International Federation of Gynecology and Obstetrics. SubCommittee on Perinatal Epidemiology and Health Statistics. Report on the methodology of measurement and recording of infant growth in the perinatal period. London: FIGO, 1986.

12 Robinson HP. Sonar measurement of fetal crown-rump length as means of assessing maturity in first trimester of pregnancy. $B M F$ 1973; 4: 28-31.

13 Campbell S. An improved method of fetal cephalometry by ultrasound. F Obstet Gynaecol Br Cwlth 1968; 75: 568-76.

14 Jeanty P, Romero R. Obstetrical ultrasound. New York: McGraw Hill, 1984.

15 Grannum PAT. Ultrasonic measurements for diagnosis. In Gross TL, Sokol RJ, eds. Intrauterine growth retardation. $A$ practical approach. Chicago: Year Book Medical Publishers, 1989: 123-57.

16 Queenan JT, O'Brien GD, Campbell S. Ultrasound measurement of fetal limb bones. Am f Obstet Gynecol 1980; 138: 297-302.

17 Kurjac A, Breyer B. Estimation of fetal weight by ultrasonic abdominometry. Am $\mathcal{F}$ Obstet Gynecol 1976; 125: 962-5.

18 Count EW. Growth pattern of the human physique: an approach to kinetic anthropometry. Part I. Hum Biol 1943; 15: 1-32.

19 Rossavik IK, Deter RL. Mathematical modelling of fetal growth: I. Basic principles. $\mathcal{F}$ Clin Ultrasound 1984; 12 529-33.

20 Wiener SN, Flynn MJ, Kennedy AW, Bonk F. A composite curve of ultrasonic biparietal diameters for estimating gestational age. Radiology 1977; 122: 581-3.

21 Milani S. Modelling kinetics of somatic growth. In: DiBacco M, Pacciani E, Borgognini-Tarli S, eds. DiBacco M, Pacciani E, Borgognini-Tarli S, eds. Statistical tools in human biology. London:

22 Berkey CS, Laird NM. Nonlinear growth curve analysis: estimating the population parameters. Ann Hum Bio 1986; 13: 111-28.

23 Erikssen PS, Secher NJ, Weis-Bentzon M. Normal growth of the fetal biparietal diameter and the abdominal diameter in a longitudinal study. Acta Obstet Gynecol Scand 1985; 64: 65-70.

24 Jeanty P, Cousaert E, Hobbins JC, Tack B, Bracken M, Cantraine F. A longitudinal study of fetal head biometry. Am $\mathcal{F}$ Perinatol 1984; 2: 118-28.

25 Munjanja SP, Masona D, Masvikeni S. Fetal biparietal diameter and head circumference measurements: results of a longitudinal study in Zimbabwe. Int f Gynecol Obstet of a longitudinal

26 Fescina RH, Ucieda FJ, Cordano MC, Nieto F, Tenzer SM, Lopez R. Ultrasonic patterns of intrauterine fetal growth in a Latin American country. Early Hum Dev 1982; 6: 239-48.

27 Deter RL, Harrist RB, Hadlock FP, Poindexter AN Longitudinal studies of fetal growth with the use of dynamic image ultrasonography. Am $\mathcal{f}$ Obstet Gynecol 1982; 143: 545-54

28 Deter RL, Harrist RB. Growth standards for anatomic measurements and growth rates derived from longitudinal studies of normal fetal growth. $\mathcal{F}$ Clin Ultrasound 1992; 20: studies

29 Dobbing J, Sands J. Head circumference, biparietal diameter and brain growth in fetal and postnatal life. Early diameter and brain growth

30 Meire HB. Ultrasound measurement of fetal growth patterns. In: Falkner F, Tanner JM, eds. Human growth. Vol 1. New York: Plenum Press, 1986:275-90.

31 Baily NTJ. The mathematical approach to biology and medicine. XIV Series on quantitative methods of biologists and medical scientists. London: Wiley, 1970.

32 Brons JTJ, van Geijn HP, Bezemer PD, Nauta JPJ, Arts NF. The fetal skeleton; ultrasonographic evaluation of the normal growth. Eur f Obstet Gynaecol Reprod Biol 1990; 34: 21-36.

33 Tanner JM. Foetus into man. 2nd edn. Welwyn Garden City: Castlemead Publications, 1989.

34 Murao F, Senoh D, Takamiya O, Yamamoto K, Hasegawa $\mathrm{K}$, Kitao $M$. Ultrasonic evaluation of liver development in the fetus in utero. Gynecol Obstet Invest 1989; 28: 198-201.

35 Micheli JL, Pfister R, Schutz Y, Calame A, Jequier E. Skinfold thickness and body composition during early postnatal growth in low birthweight infants. Dev Physiopathol Clin 1991; 2: 113-23.

36 Singer DB, Sung CJ, Wigglesworth JS. Fetal growth and maturation with standards for body and organ development. In: Wigglesworth JS, Singer DB, eds. Textbook of fetal and perinatal pathology. Vol 1. London: Blackwell Scientific Publications, 1991:11-47.

37 Vaucher YE, Harrison GG, Udall JN, Morrow G. Skinfold thickness in North American infants 24-41 weeks thickness in North American infa

38 Poissonnet CM, Burdi AR, Garn SM. The chronology of adipose tissue appearance and distribution in the human fetus. Early Hum Dev 1984; 10: 1-11.

39 Hata T, Deter RL. A review of fetal organ measurements obtained with ultrasound: normal growth. $f$ Clin Ultrasound 1992; 20: 155-74.

40 Ogata ES. Carbohydrate metabolism in the fetus and neonate and altered neonatal glucoregulation. Ped Clin North Am 1986; 33: 25-45.

41 Brar HS, Rutherford SE. Classification of intrauterine growth retardation. Semin Perinatol 1988; 12: 2-10.

42 Fearn T. A bayesian approach to growth curves. Biometrika 1975; 62: 89-100. 